\title{
Variação Pluviométrica e Parâmetros Físico-químicos da Água Tratada para Distribuição em Porto Velho, Rondônia.
}

\section{Variation Pluviometric, Chemical And Physical Parameters Of Water Treated For Distribution In Porto Velho, Rondônia.}

\begin{abstract}
Márcia Bay
Resumo: O regime pluviométrico do município de Porto Velho capital de Rondônia (Amazônia brasileira) é bastante regular, variando apenas nos três meses mais secos de cada ano (Aw na classificação de Köppen). A qualidade da água neste município é atualmente um fator preocupante, tendo em vista o rápido crescimento demográfico ocorrido nos últimos quatro anos. Este fato se deve aos investimentos federais em um grande complexo hidrelétrico construído no Rio Madeira em Porto Velho (Usinas de Jirau e Santo Antônio). Quanto maior o consumo de água, maior a quantidade de água a ser tratada para a distribuição. A adição de produtos químicos como sulfato de alumínio $\left(\mathrm{Al}_{2}\left(\mathrm{SO}_{4}\right)_{3}\right)$ e cloro $\left(\mathrm{Cl}_{2}\right)$ para o tratamento da água é feita com base em normas técnicas e a quantidade dos mesmos varia de acordo com o período do ano e o regime pluviométrico local. A variação da quantidade de chuva pode interferir nos valores de parâmetros básicos como $\mathrm{pH}$, Turbidez e Cloro residual da água, sendo a proposta deste trabalho, fazer o comparativo do comportamento destes parâmetros com base na variação das chuvas em Porto Velho. Os dados mostraram uma regularidade nos parâmetros (pH, turbidez e cloro residual) analisados nos meses com menor precipitação.
\end{abstract}

Palavras-chaves: Tratamento de água, pluviosidade, crescimento populacional, precipitação.

Abstract: The rainfall in the city of Porto Velho capital of Rondônia (Brazilian Amazon) is fairly regular, varying only in the three driest months of each year (in the Köppen Aw). Water quality in this municipality is currently a concern in view of the rapid population growth occurred in the last four years. This is due to the federal investments in a large hydroelectric complex built on the Madeira River in Porto Velho (Plants Jirau and San Antonio) .The higher the water, the greater the amount of water to be treated for distribution. The addition of chemicals such as aluminum sulfate (Al2 (SO4)3) and chlorine (Cl2 ) for the treatment of water is made based on technical requirements and the amount thereof varies depending on the time of year, and rainfall site. Varying the amount of rain can interfere with the basic parameter values such as $\mathrm{pH}$, turbidity and residual chlorine in water, the purpose of this work, make the comparative behavior of these parameters based on the variation of rainfall in Porto Velho. The data showed a regularity in the parameters $(\mathrm{pH}$, turbidity and residual chlorine) analyzed the months with lower precipitation

Key words: Treatment of water, rainfall, population growth, rainfall.

\footnotetext{
*Autor para correspondência

Recebido para publicação em 11/06/2015; aprovado em 10/07/2015

Instituto Federal de Ciência e Tecnologia de Rondônia. E-mail: marcia.bay@ifro.edu.br
} 


\section{INTRODUÇÃO}

Com o aumento das aglomerações humanas e com a respectiva elevação do consumo da água o homem passou a executar grandes obras destinadas à captação, transporte e armazenamento deste líquido e também a desenvolver técnicas de tratamento interferindo assim no ciclo hidrológico e gerando um ciclo artificial da água (Andrade e Azevedo, 2013). Nos centros urbanos a necessidade de infraestrutura adequada para o funcionamento da sociedade de forma satisfatória é necessário à instalação de serviços básicos como a implantação da rede de distribuição de energia elétrica, rede de distribuição de água entre outros serviços como estradas etc (ANDRADE e AZEVEDO, 2013).

No que diz respeito à distribuição de água tratada de forma adequada existem necessidades muito evidentes do uso de tecnologias mais avançadas visando aumentar a qualidade da água como também melhorar a qualidade de vida dos consumidores (ALMEIDA et al., 2012). A Companhia de Águas e Esgotos do Estado de Rondônia - CAERD, Regional de Porto Velho - Rondônia, desempenha um papel muito importante no tratamento e distribuição de água, principalmente no que se refere à melhoria da qualidade desta, como também nos avanços em relação à distribuição de forma controlada, evitando-se, assim, o desperdício, sendo o maior problema em relação aos gastos desnecessários.

A companhia de água e esgotos de Rondônia foi criada pelo Decreto-Lei no 490, de 4 de março de 1969 (Rondônia, 1969). O Poder Executivo ficou autorizado a constituir sociedades de economia mista destinadas a coordenar o planejamento, executar, operar e explorar os serviços públicos de saneamento básico (abastecimento de água e esgotos sanitários) nos Territórios Federais do Amapá, Rondônia e Roraima, mediante convênios com os municípios (RONDÔNIA, 1969). A CAERD atende aos padrões de potabilidade estabelecidos pela Portaria 518 de março de 2004 do Ministério da Saúde, que define uma série de parâmetros físicos, químicos e microbiológicos, que devem ser analisados para garantir a qualidade da água (SOUZA, 2012). O controle de qualidade é realizado através de análises executadas em laboratório próprio, com pessoal especializado e os resultados estão disponíveis na conta mensal de serviços de água e esgoto (SOUZA, 2012). A água tratada é um produto industrial que exige altos investimentos para a sua produção, tratamento, reservação, distribuição e controle de qualidade. Para garantir a quantidade e qualidade dentro dos padrões estabelecidos pela Organização Mundial de Saúde OMS, a CAERD exerce um rigoroso controle (SOUZA, 2012).

A capital de Rondônia, Porto Velho, vem desde o final da década de 2000 passando por sérios problemas de ordem urbana, como a chegada das usinas de Jirau e Santo Antônio no Rio Madeira, que trouxeram consigo uma grande quantidade de trabalhadores oriundos de diversas partes do Brasil (IBGE, 2013). Houve também, um aumento significativo da população em Porto Velho para de cerca de 600 mil habitantes entre 2000 e 2013 (IBGE, 2013).

A estação de Tratamento de Água (ETA) de Porto Velho tem como objetivo a captação e tratamento de água do Rio Madeira para a distribuição à população da cidade, o qual sofreu uma alteração no local de captação com a construção da Usina Hidrelétrica de Santo Antônio (ARANDA, 2012).
O ponto de captação de água da Companhia de Abastecimento de Água e Esgoto de Rondônia (CAERD) de Porto Velho começou a ficar atolado na lama em consequência da construção da Usina Hidrelétrica de Santo Antônio e o abastecimento da população da cidade estava sendo escalonado. De acordo com a diretora técnica da CAERD, a captação que era de 650 litros por segundo diminuiu para 400 litros por segundo (ARANDA, 2012). Além disso, a turbidez da água obriga a paradas contínuas para a limpeza dos oito filtros da estação de tratamento e ao aumento do volume de produtos utilizados para tornar a água potável, prejuízos que viam sendo acumulados pela empresa, já que não pode ser repassada para os consumidores, por isso, a mudança do local de captação para a ETA da CAERD (ARANDA, 2012).

Segundo Mendonça (2007) a variabilidade pluviométrica no Brasil, assim como sua distribuição e atuação estão diretamente ligadas à sazonalidade das frentes polar atlântica (FPA). Na região amazônica há um regime pluviométrico característico, tendo em vista que no chamado inverno amazônico (verão no hemisfério sul), sendo os maiores índices pluviométricos registrados (novembro a abril) e os meses mais secos e com menor carga pluvial vão de maio a outubro. É sabido que conforme temos um aumento do volume de chuvas aumenta também o fluxo de sedimentos nos rios. O Rio Madeira em Rondônia, ponto de captação de água para tratamento e distribuição da água em Porto Velho, tem um alto fluxo de sedimentos transportados (FISCH et al., 1998).

A grande quantidade de materiais diluídos e suspensos na água alteram visivelmente dois parâmetros de qualidade, a turbidez e a cor. A presença em grande quantidade de argilas e silte nas águas do Rio Madeira aumenta conforme a época do ano. Para Filizola e Guyot (2011) os rios estão no centro do ciclo dos elementos no que se refere ao transporte de matéria sublevada dos continentes para os oceanos, respondendo rapidamente às condições existentes no meio, em questão de meses para transporte em solução e em questão de anos para transporte em suspensão. Ainda segundo Filizola e Guyot (2011) os rios são particularmente sensíveis à todos os tipos de mudanças no ambiente, inclusive climáticas. As maiores taxas de erosão são percebidas em bacias hidrográficas situadas nas zonas tropicais e em especial aquelas que drenam cadeias montanhosas ativas como os Andes, por exemplo, (PINET e SOURIAU 1988, MILLIMAN e SYVITZKI 1992, SUMMERFIELD e HULTON 1994). Stenberg $(1975,1995)$ apud Filizola e Guyot (2011) afirmam que no caso da bacia amazônica as ações antrópicas sem controle interferem diretamente nos processos de erosão. Essa influência pode estar refletida na variação pluviométrica, que por sua vez altera o volume de sedimentos, que por sua vez interfere nos processos de tratamento da água a ser distribuída (FISCH et al., 1998).

Assim o objetivo deste trabalho é analisar se há uma relação direta ou indireta entre a pluviosidade e os parâmetros de qualidade da água que serão analisados: $\mathrm{pH}$, turbidez e cloro residual.

\section{MATERIAL E MÉTODOS}

Em visita a Estação de Tratamento de Água em Porto Velho (Figura 1), onde também se localiza o ponto de captação conseguimos uma planilha contendo os valores dos 
seguintes parâmetros de qualidade da água para o município de Porto Velho: Turbidez, pH e Cloro Residual, no período janeiro de 2010 a julho de 2013.

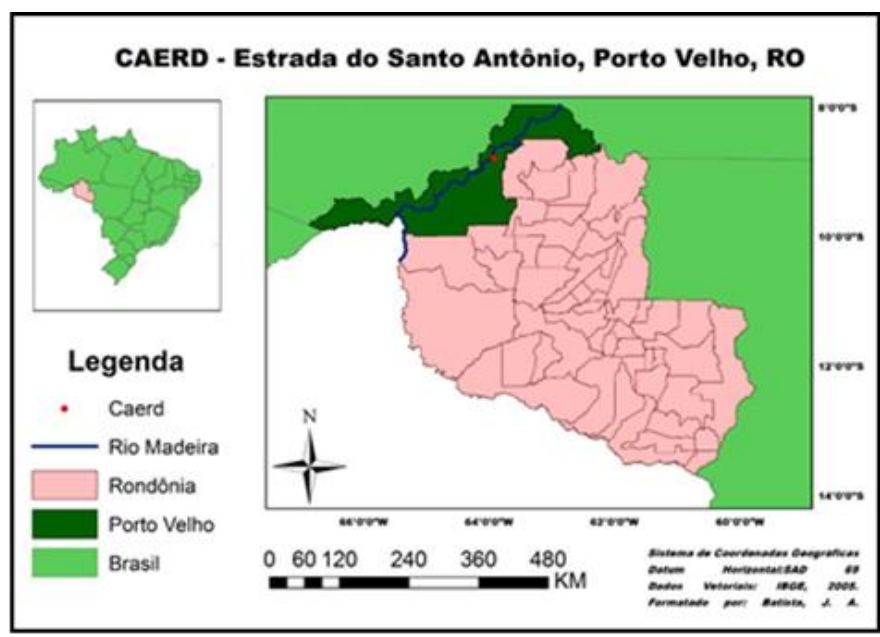

Figura 1: Localização da ETA-CAERD, Porto Velho. Cartografia:

J.A. Batista.

Estes dados foram cruzados com gráficos de pluviosidade disponibilizados pelo Instituto Nacional de Meteorologia (INMET) referente ao mesmo recorte temporal dos dados disponibilizados pela CAERD.

Utilizando o método hipotético-dedutivo onde através do qual construímos uma teoria que fórmula hipóteses a partir dos quais os resultados obtidos podem ser deduzidos (SPOSITO, 2004), verificamos a relação da pluviosidade e carga de sedimentos e sua influência na possível alteração dos parâmetros de qualidade da água supracitados. Tomamos os meses de junho, julho e agosto como referência para os meses mais secos; e os meses de dezembro, janeiro e fevereiro como os períodos de maior descarga pluvial. A Figura 2 mostra o Rio Madeira no período do verão amazônico que mesmo sem a presença de chuvas a água permanece com a cor escura e

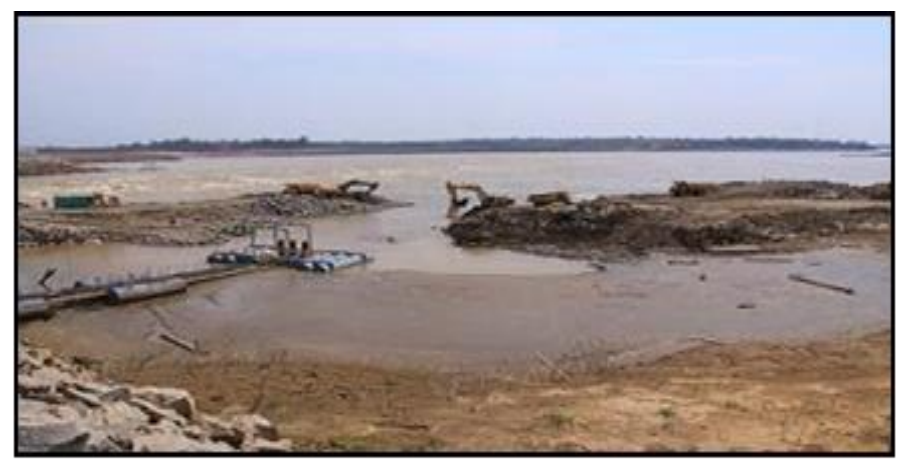

presença de muitos sedimentos.

Figura 2: Ponto de capitação de água pela CAERD no rio Madeira. Imagem: Jota Gomes, 2014

\section{RESULTADOS E DISCUSSÃO}

Comparando os dados meteorológicos disponíveis no site do INMET relativos à pluviosidade em Porto Velho e os gráficos confeccionados através dos valores disponibilizados pela CAERD conseguimos visualizar que a quantidade de chuva e os períodos mais chuvosos e menos chuvosos interferem nos valores dos parâmetros de qualidade da água: Turbidez, pH e cloro residual. A figura 3 mostra a quantidade chuvas em milímetros (mm) no município de Porto Velho no ano de 2010, mostrando claramente os períodos de menor índice pluviométrico no ano, onde os meses de junho, julho e agosto apresentaram uma pluviosidade baixa (normal para a região) variando entre $50 \mathrm{~mm}$ em junho e $35 \mathrm{~mm}$ em agosto (mês mais seco).

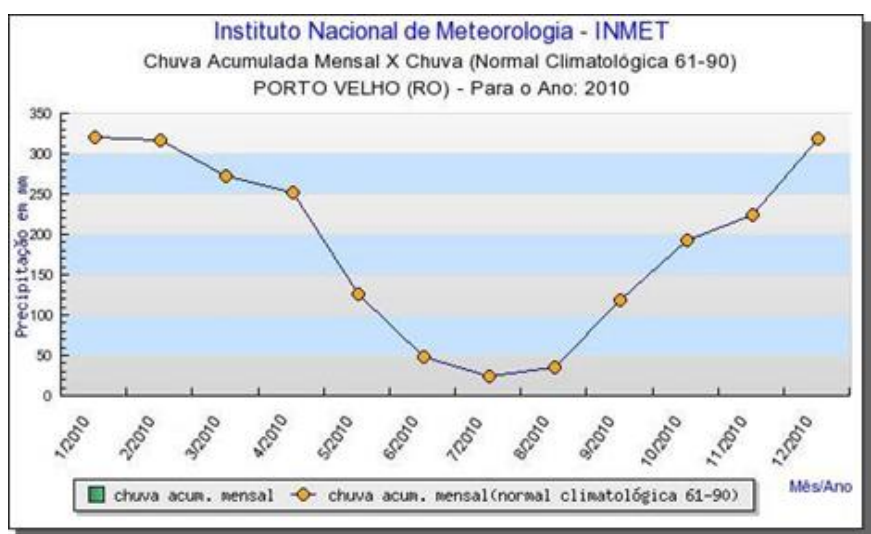

Figura 3: Pluviosidade em Porto Velho no ano de 2010. Fonte: INMET, 2013

A Figura 4 apresenta os dados referentes aos parâmetros físico-químicos da água, disponibilizados pela CAERD para o ano de 2010.

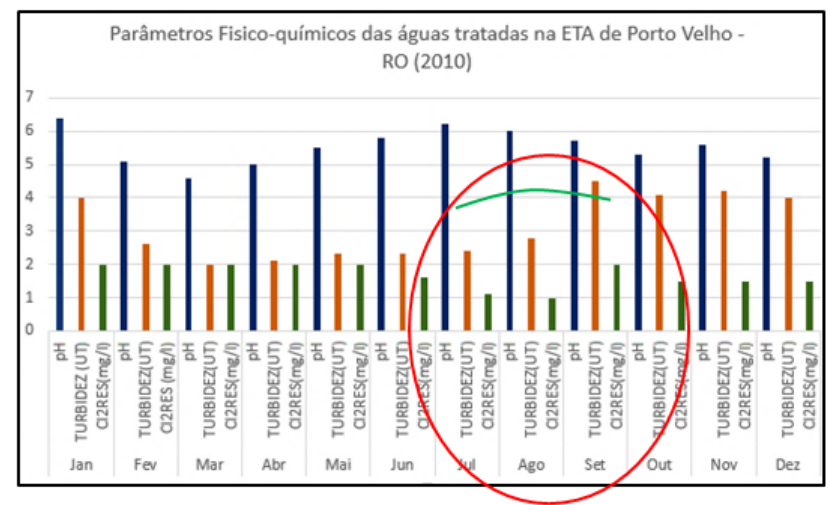

Figura 4: Parâmetros Físico-químicos das águas tratadas na ETA de Porto Velho, RO 2010.

Fonte: CAERD, 2013

Demos ênfase neste caso, aos meses de junho, julho e agosto, não só por serem os meses mais secos, mas por apresentarem nitidamente ao visualizar o gráfico, as maiores variações nos parâmetros analisados, principalmente no tocante a turbidez e ao cloro residual. $\mathrm{O}$ pH se mantém relativamente estável sem grandes alterações (6,2 em junho, 6,0 em julho e 5,7 em agosto), no tocante à turbidez é notável um aumento das UT's (2,3 em junho, 2,4 em julho e 2,8 em agosto) no período citado, o que é algo anormal, tendo em vista que nos períodos mais secos a turbidez geralmente se apresenta mais baixa devido à redução no transporte de sedimentos; o cloro residual livre se apresenta dentro da faixa tolerável pelo CONAMA (Conselho Nacional de Meio Ambiente) que segundo o manual da CETESB (2012) deve se apresentar com um valor máximo de $5 \mathrm{mg} . \mathrm{L}^{-1} \mathrm{Cl}_{2}(1,6 \mathrm{em}$ junho, 1,1 em julho e 1,0 em agosto).

As Figuras 5 e 6 apresentam respectivamente os dados pluviométricos e os parâmetros físico-químicos da água tratada em Porto Velho, RO para o ano de 2011. 


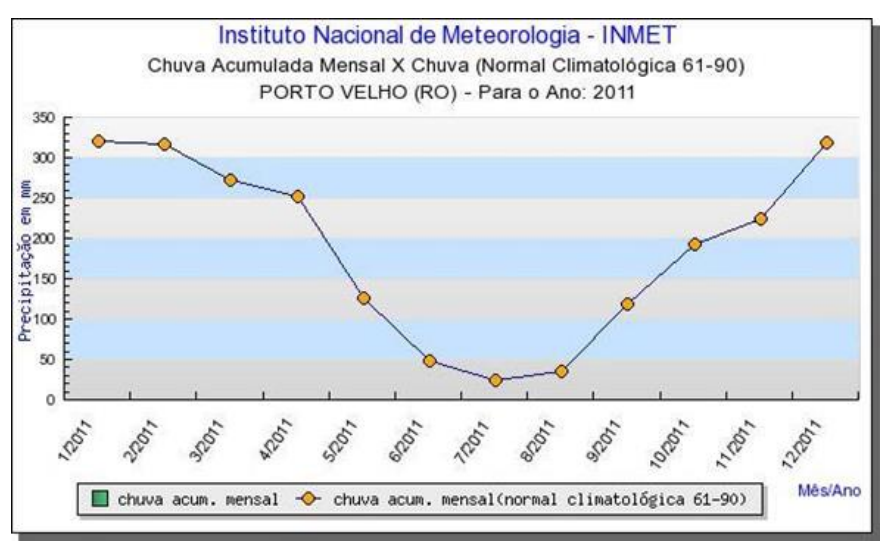

Figura 5: Pluviosidade em Porto Velho no ano de 2011.

Fonte: INMET, 2013

Novamente estão em destaque os meses de junho, julho e agosto, sendo que para o referido ano temos algumas diferenças entre os padrões apresentados para o ano de 2010 referentes aos parâmetros apresentados (os mesmos do ano anterior). A pluviosidade nos meses de junho a agosto em 2011 declinou variando entre $50 \mathrm{~mm}$ para junho, cerca de 30 mm para o mês de julho e cerca $35 \mathrm{~mm}$ para o mês de agosto (geralmente o mês que chove menos é agosto). Vemos na Figura 6 para os meses em destaque que não há uma variação acentuada em todos os parâmetros. Desta vez a variação que se destaca é no parâmetro pH saindo de 5,1 em junho para 5,7 em julho e permanecendo com mesmo valor no mês de agosto. Segundo informação de funcionários da CAERD, o $\mathrm{pH}$ varia devido a adição de substâncias químicas como sulfato de alumínio colocadas na água para a coagulação de elementos indesejáveis ao bom tratamento. A turbidez também apresentou diferenças entre os valores (2,6 em junho, 3,4 em julho e 3,2 em agosto), porém não ultrapassando os valores permitidos pelo CONAMA 357/05 que são de 5 UNT. Quanto ao cloro residual $\left(\mathrm{Cl}_{2}\right)$ não houve alteração, mantendo-se em $2 \mathrm{mg} . \mathrm{L}^{-1}$ nos três meses, dentro do padrão CONAMA que é de $5 \mathrm{mg} . \mathrm{L}^{-1}$.

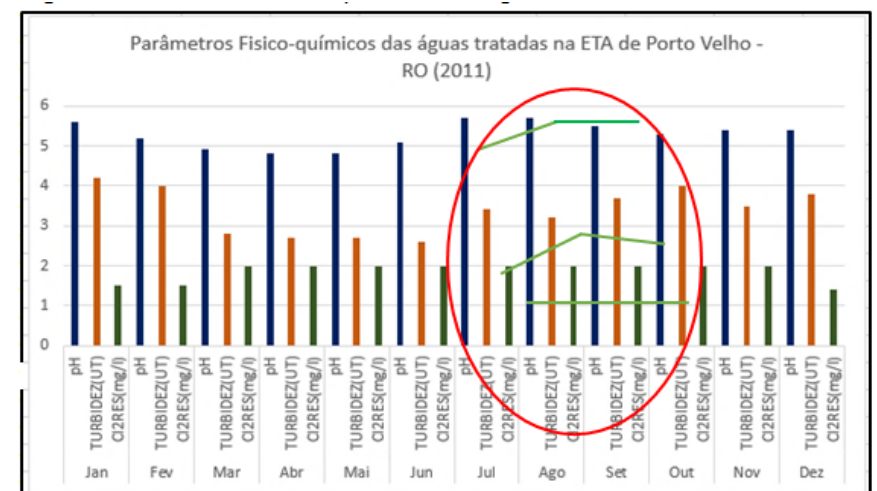

Figura 6: Parâmetros Físico-químicos das águas tratadas na ETA de Porto Velho, RO, 2011

Fonte: CAERD, 2013

Para 2012 o índice pluviométrico (Figura 7), para os meses avaliados, variou normalmente (entre $50 \mathrm{~mm}$ no mês de junho, cerca de $20 \mathrm{~mm}$ no mês de julho e aproximadamente $35 \mathrm{~mm}$ para o mês de agosto), permanecendo a tendência de pouca variação também dos parâmetros físico-químicos analisados.

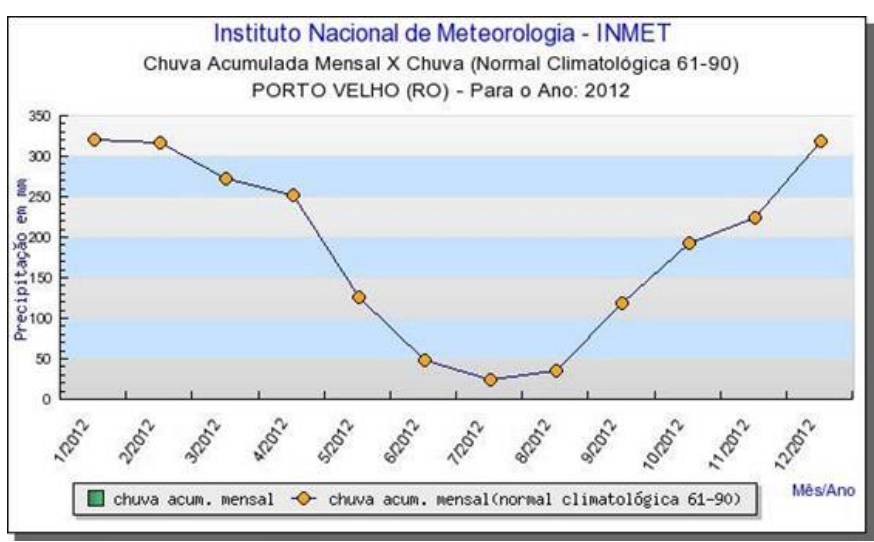

Figura 7: Pluviosidade em Porto Velho no ano de 2012. Fonte: INMET, 2013

Para o $\mathrm{pH}$ seguiu-se uma crescente saindo de 5,1 em junho, para 5,2 e 5,3 respectivamente para julho e agosto ficando um pouco abaixo dos valores estabelecidos pelo CONAMA que devem estar entre 6 e 8 . A turbidez aumentou de 2,6 UNT em junho para 3,0 UNT em julho aumentando conforme diminuiu o pH e 2,8 UNT em agosto. O cloro residual variou em $0,5 \mathrm{mg} . \mathrm{L}^{-1}$, saindo de $1,5 \mathrm{mg} . \mathrm{L}^{-1}$ em junho para $2,0 \mathrm{mg} . \mathrm{L}^{-1}$ em julho e retornando para $1,5 \mathrm{mg} . \mathrm{L}^{-1} \mathrm{em}$ agosto. Conforme mostra a figura 8 .

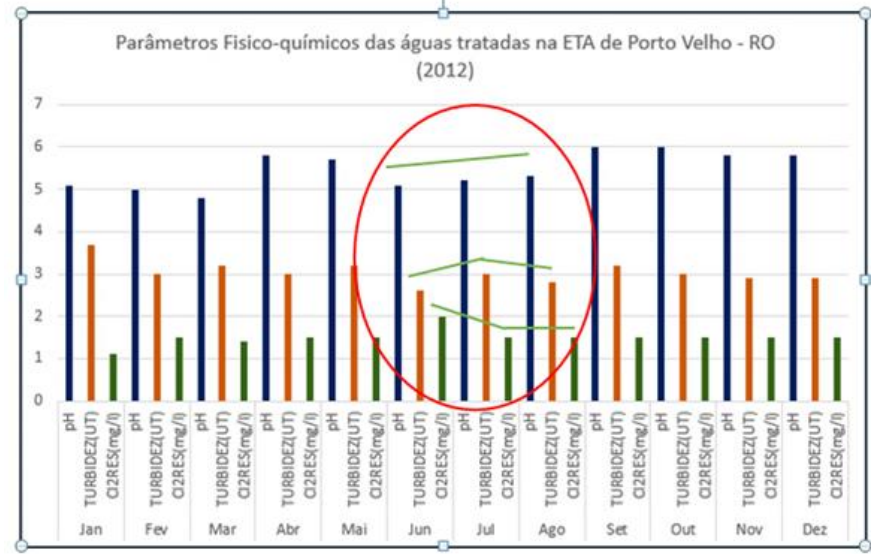

Figura 8: Parâmetros Físico-químicos das águas tratadas na ETA de Porto Velho, RO, 2012

A comparação deste recorte temporal (2010, 2011 e 2012) nos mostrou que não houve variação dos parâmetros avaliados dentro do período mais seco (menores índices de precipitação pluviométrica) para Porto Velho que vai de junho a agosto nos anos de 2010, 2011 e 2012. De início relacionamos a possibilidade de a quantidade de sedimentos transportados no Rio Madeira seria fator influente no tratamento e na posterior qualidade da água disponível para o consumo em na capital rondoniense.

A verificação destes parâmetros ( $\mathrm{pH}$, Turbidez e Cloro residual) está diretamente ligada a disponibilidade de dados por parte do órgão responsável pelo tratamento da água no município de Porto Velho que é a CAERD e tendo em vista que temos apenas três meses de seca constante em relação à nove meses de chuvas de alta e média magnitude escolhemos junho, julho e agosto para serem analisados. Este trabalho não teve a pretensão de comparar e correlacionar a influência da relação entre os três parâmetros aqui discutidos, mas sim apresentar um recorte temporal do comportamento de tais parâmetros no período mais seco em Porto Velho. 


\section{CONCLUSÕES}

De acordo com as análises dos dados percebe-se uma regularidade em relação às normas e portarias estabelecidas pelo Conselho Nacional de Meio Ambiente o CONAMA, sem apresentar alterações ou inconformidades legais para os parâmetros no período analisado.

\section{REFERÊNCIAS BIBLIOGRÁFICAS}

ALMEIDA, M. A. B.; GUTIERREZ, G. L.; MARQUES, R. Qualidade de vida: definição, conceitos e interfaces com outras áreas de pesquisa. São Paulo: Editora Escola de Artes, Ciências e Humanidades - EACH/USP, 2012. 142p.

ANDRADE, F. L. S.; AZEVEDO, A. K. N. A influência do aumento de nitrato na saúde das populações de baixa renda econômica. In: Simpósio de Ciências Biológicas, VI, 2013, São Paulo. Anais Eletrônicos... Universidade de Campinas, Campinas, São Paulo, Brasil, 2013.

ARANDA, A. Construção de hidrelétrica de Santo Antônio obriga Caerd a escalonar abastecimento de água. Disponível em <http://www.amazoniadagente.com.br/?p=10470>, Acesso em 24 Jan. 2015.

AYERS, R. S.;WESTCOT, D. W. A qualidade da água na agricultura. Tradução de h.R. Ghei. J.F.v Damasceno. 2.ed. Campina Grande: UFPB, 1999. 153p Estudos FAO Irrigação e Drenagem, 29 revisado.

BAGLEY, C.V.; KOTUBY-AMACHER, J.FARREL-POE, K.. Utah State University Extension. 7p. 1997.

BRAUL, L.; KIRYCHUK, B. Water quality and cattle. Agriculture and Agri-Food. 6p. 2001.

DIAS,N.S.;CHEYI, H.R.; DUARTE, S.N. Prevenção, manejo e recuperação dos solos efetados por sais. Piracicaba: ESALQ/USP/LER, 2003 (Série Didática, 013).

FISCH, G.; MARENGO, J. A.; NOBRE, C. A. Uma revisão geral sobre o clima da Amazônia. Acta Amazônica, V. 28. N. 2, p: 101-126, 1998.
INSTITUTO BRASILEIRO DE GEOGRAFIA E ESTATÍSTICA - IBGE, Diretoria de Pesquisas, Coordenação de População e Indicadores Sociais. Estimativas da população residente com data de referência 1o de julho de 2014. Diário Oficial da União. 28 Ago. 2014. Disponível em <http://www.ibge.gov.br/home/estatistica/populacao/esti mativa2014/default.shtm>. Acesso em: 23 Jan. 2015.

LACERDA, C.F. Integração salinidade $\mathbf{x}$ nutrição mineral. In: Nogueira, R. J. M. C.; Araújo, E. de L.; Willadino, L. g.; Cavalcante, U. M. T.(ed). Estresses ambientais: Danos e benefícios em plantas. Recife: UFPE, 2005, P.127-137.

LIMA, L. A. Efeitos de sais no solo e na planta. In: Gheyi, H. R.; Campina Grande UFPB; SBEA, 1997. p113-136.

PIZARRO, F. Riegos localizados de alto frequência (RLAF). Goteo, microaspersión y exudación. 3.ed. ver. Y amp. Madrid: Mundi-Prensa . 1996. 513p.

QUEIROZ, J.E. Avaliação e monitoramento da salinidade do solo. In: GHEYI H R; DIAS N S; LACERDA C F. Manejo da salinidade na agricultura: estudos básicos e aplicados. Fortaleza, INCT Sal, 2010.

RONDÔNIA, Decreto-Lei no 490, de 4 de Março de 1969, Autoriza o Poder Executivo a criar Companhias de Águas e Esgotos para os Territórios do Amapá, Rodônia e Roraima e dá outras providências. Diário Oficial, Brasília, DF, 05 março 1969, Seção 1, p. 1905.

SOUZA, R. A. Bio-físico-química de poços cacimba do centro urbano de Ouro Preto do Oeste - RO: uma análise sócio espacial. Dissertação de Mestrado. Departamento de Geografia, Fundação Universidade Federal de Rondônia, Porto Velho, Rondônia, 108f. 2012.

SPERLING, M. V. Introdução a qualidade das águas e ao tratamento de esgotos/ Marcos Von Sperling. - 3. Ed. Belo Horizonte: Departamento de Engenharia Sanitária e Ambiental; Universidade Federal de Minas Gerais; 2005.

SILVA, Í. N.; FONTES, L. O.; TAVELLA, L. B.; et al. Qualidade de água na irrigação. Agropecuária Científica no Semiárido, v.07, n 03, p. 01 - 15, 2011. 